\title{
A Mixed Regularization Method for Ill-Posed Problems
}

\author{
Hui Zheng ${ }^{1,2}$ and Wensheng Zhang ${ }^{2,3 *}$ \\ ${ }^{1}$ School of Mathematics and Statistics, Huazhong University of Science and \\ Technology, Wuhan 430074, China \\ ${ }^{2}$ LSEC, ICMSEC, Academy of Mathematics and Systems Science, Chinese Academy \\ of Sciences, Beijing 100190, China \\ ${ }^{3}$ School of Mathematical Sciences, University of Chinese Academy of Sciences, \\ Beijing 100049, China
}

Received 30 June 2017; Accepted (in revised version) 6 March 2018

\begin{abstract}
In this paper we propose a mixed regularization method for ill-posed problems. This method combines iterative regularization methods and continuous regularization methods effectively. First it applies iterative regularization methods in which there is no continuous regularization parameter to solve the normal equation of the illposed problem. Then continuous regularization methods are applied to solve its residual problem. The presented mixed regularization algorithm is a general framework. Any iterative regularization method and continuous regularization method can be combined together to construct a mixed regularization method. Our theoretical analysis shows that the new mixed regularization method is with optimal order of error estimation and can reach the optimal order under a much wider range of the regularization parameter than the continuous regularization method such as Tikhobov regularization. Moreover, the new mixed regularization method can reduce the sensitivity of the regularization parameter and improve the solution of continuous regularization methods or iterative regularization methods. This advantage is helpful when the optimal regularization parameter is hard to choose. The numerical computations illustrate the effectiveness of our new mixed regularization method.
\end{abstract}

AMS subject classifications: 65F22, 35R25, 35R30, 65N20, 65N21

Key words: Ill-posedness, continuous regularization, iterative regularization, mixed regularization, optimal order.

\section{Introduction}

Suppose that $T: X \rightarrow Y$ is a bounded linear operator, here $X$ and $Y$ are two Hilbert spaces. The inverse problem is that $y \in Y$ is known and we seek $x \in X$ such that $T x=$ $y$. Ill-posedness always appears in inverse problems if $T$ is compact, i.e., the solution $x$

${ }^{*}$ Corresponding author. Email address: zws@lsec.cc.ac.cn (W. S. Zhang) 
may not satisfy existence, uniqueness or continuity. Even if we can ensure the existence and uniqueness of the solution $x$ in some sense, the non-continuity still leads to difficulty in computations. The non-continuity means that the solution $x$ is very sensitive to any perturbation of the right-hand side $y$, i.e., small perturbations of $y$ can produce arbitrarily large perturbations of the solution $x$. The perturbation of $y$ usually represents the noises in the data. The method to overcome the ill-posedness is known as the regularization $[3,14,42]$. Generally speaking, the regularization methods can be classified into two categories: continuous regularization methods and iterative regularization methods. In this article we try to combine the two types of regularization methods together and we name it the mixed regularization method.

The typical continuous regularization methods include the truncated singular value decomposition (TSVD) method and Tikhonov regularization method. Among these methods, Tikhonov regularization method is the widely used method. It is proposed firstly by Tikhonov [37, 38] in 1963 and then is applied in solving ill-posed problems [39, 40]. Other methods include the stationary and non-stationary iterated Tikhonov method and so on [13]. In the continuous regularization methods, one important step is the choice of the regularization parameter. If the parameter is too small, the ill-posedness of the original problem can not be overcome effectively and the error from the ill-posedness is dominated. On the contrary, if the parameter is too large, the consistency between the original problem and the regularized problem become large and the error from this factor is dominated. Therefore, it is crucial to balance these two kinds of errors. Many methods on how to choose the optimal parameter are investigated, for example, the discrepancy principle $[18,27,33,41,44]$, the L-curve criterion $[15,16]$, the generalized crossvalidation $[1,6,43]$, and so on. However, how to choose a optimal regularization parameter effectively is still worth studying in solving real problems when we have little knowledge about the exact solution.

Iterative regularization methods are another type of frequently-used regularization methods. In iterative regularization methods, the stopping index is regularization parameter and there is no continuous regularization parameter. For well-posed problems, the iterative solution usually convergences to the exact solution as the number of iterations increases. However, for ill-posed problems, there exists a phenomenon called semiconvergence, i.e., the iterative solution converges to the exact solution in first several iterations, but it goes away from the exact solution after a certain step. Thus, some stopping rules must be used so that the iteration can stop at certain iteration which is closest to the exact solution. The popular iterative regularization methods include the Landweber method $[2,9,12,21,26,30,32]$, the conjugate gradient (CG) method $[5,10,11,25,28,29]$ and so on [14].

In iterative regularization methods, the number of iterations or the stopping index is a regularization parameter and this parameter is on the set of natural number. In contrast, the regularization parameter in continuous regularization methods is on the set of real numbers. Therefore, in iterative regularization methods, the parameter (i.e., the number of iterations) can not be chosen as precisely as that in continuous regularization methods. For example, the iterative solution usually does not approximate the exact solution 
enough after some iteration, but goes away from the direction just after the next iteration. Between these two iterative solutions, we can not obtain the other numerical solutions which are probably more precise. Although some iterative regularization methods, such as Landweber methods and conjugate gradient methods, can attain the optimal order under some stopping rules, the iterative solutions usually could be improved further.

In order to improve the iterative solution, we propose a mixed regularization method in this paper. First we solve the original problem with an iterative regularization method. Then we solve the residual equation with a continuous regularization method. Finally we add the solution of the residual equation to the original iterative solution. The solution of this mixed regularization method can improve the solution from iteration regularization methods. This fact is proved by our theoretical analysis (Theorem 3.2) and is confirmed by the numerical computations.

The new mixed regularization method also can be considered as an improvement of continuous regularization methods. In continuous regularization methods, the choice of the parameter is crucial because the accuracy of solutions depends on the regularization parameter sensitively. Our theory (Theorem 3.1) shows that the new mixed regularization method reduces the sensitivity of the regularization parameter. Specifically speaking, the mixed regularization methods can reach the optimal order with a much wider range of the regularization parameter than the continuous regularization method. This theorem is also confirmed by our numerical computations.

Another advantage of our mixed regularization method is that it is a general algorithm framework. Any iterative regularization method and any continuous regularization method can be combined together to construct a mixed regularization method. The mixed method All these mixed regularization methods can be analyzed in our theoretical framework.

The rest of this article is arranged as follows. In Section 2, we discuss the regularization methods for ill-posed problems based on the functional framework of [4]. In Section 3, we present our new mixed regularization method and give the theoretical analysis. In Section 4, we present numerical results to illustrate the theory in Section 3. Finally we give a conclusion in Section 5.

\section{Regularization methods}

Consider the equation

$$
T x=y,
$$

where $X$ and $Y$ are two Hilbert spaces and $T: X \rightarrow Y$ is a bounded linear operator. Because of the ill-posedness, the solution $x$ of (2.1) possibly does not satisfy existence or uniqueness. However, we can determine a unique $x \in X$ in some senses. We call $x \in X$ the least-squares solution of (2.1) if

$$
\|T x-y\|=\inf \{\|T z-y\| \mid z \in X\}
$$


and we call $x \in X$ the best-approximate solution of (2.1) if

$$
\|x\|=\inf \{\|z\| \mid z \text { is least-squares solution of (2.1) }\} \text {. }
$$

By the theory of Moore-Penrose generalized inverse [4], we can define the Moore-Penrose generalized inverse $T^{\dagger}$ of operator $T$ on the domain

$$
\mathscr{D}\left(T^{\dagger}\right)=\mathscr{R}(T)+\mathscr{R}(T)^{\perp}
$$

then we obtain a unique $x^{\dagger}=T^{\dagger} y$ for $y \in \mathscr{D}\left(T^{\dagger}\right)$. It can be shown that, for $y \in \mathscr{D}\left(T^{\dagger}\right)$, (2.1) has a unique best-approximate solution and this solution is exactly the Moore-Penrose generalized inverse

$$
x^{\dagger}=T^{\dagger} y .
$$

Therefore, our goal is to find a numerical solution $x^{\text {sol }}$ which is as close as possible to $x^{\dagger}$. That is, we want $\left\|x^{\text {sol }}-x^{\dagger}\right\|$ to be as small as possible.

In most practical problems, the observed data $y$ contains noise, That is to say, the exact right-hand side $y^{\text {exa }}=T x^{\dagger}$ can not be attained. We assume there is a perturbed right-hand side $y^{\delta}$ with a noise level $\delta$, i.e.,

$$
\left\|y^{\delta}-y^{\text {exa }}\right\| \leqslant \delta
$$

Thus, in fact we obtain our solution $x^{\text {sol }}$ through the equation

$$
T x=y^{\delta} .
$$

In order to analyze the error $\left\|x^{\text {sol }}-x^{\dagger}\right\|$ theoretically, we introduce some notation of functional calculus. Using the notation of functional calculus, we introduce a set

$$
X_{\mu, \rho}=\left\{x \mid x=\left(T^{*} T\right)^{\mu} w,\|w\| \leqslant \rho\right\},
$$

where $\mu>0$ and $T^{*}$ is the adjoint operator of $T$. By classical theory in inverse problems $[4,20]$, for $x^{\dagger} \in X_{\mu, \rho}$, any method can not ensure an error better than

$$
\left\|x^{\mathrm{sol}}-x^{\dagger}\right\| \leqslant \delta^{\frac{2 \mu}{2 \mu+1}} \rho^{\frac{1}{2 \mu+1}}
$$

Therefore, if a method ensures the error

$$
\left\|x^{\mathrm{sol}}-x^{\dagger}\right\| \leqslant c \delta^{\frac{2 \mu}{2 \mu+1}} \rho^{\frac{1}{2 \mu+1}}
$$

we call this method is with optimal order in $X_{\mu, \rho}$. Notice that $c$ in (2.10) denotes a constant and we will use the similar notation in the following. 


\subsection{Continuous regularization methods}

In this subsection, we analyze continuous regularization methods under the framework in [4]. They are convenient and beneficial for our analysis in the mixed regularization method in Section 3.

Writing (2.7) in form of normal equation

$$
T^{*} T x=T^{*} y^{\delta} .
$$

Using the exact right-hand side $y^{\text {exa }}$ instead of $y^{\delta}$, we solve (2.11) as

$$
x^{\dagger}=\left(T^{*} T\right)^{-1} T^{*} y^{\text {exa }} .
$$

However, operator $\left(T^{*} T\right)^{-1}$ is usually not well-posed for a perturbed right-hand side $y^{\delta}$. In continuous regularization methods, we introduce a function $g_{\alpha}(\lambda)$, which is associated with $\alpha$, to express the solution. Here $\alpha$ is the regularization parameter. Then the regularized solution $x^{\text {sol }}$ can be formally written as $[4,22,34]$

$$
x^{\mathrm{sol}}=g_{\alpha}\left(T^{*} T\right) T^{*} y^{\delta} .
$$

If $g_{\alpha}(\lambda)$ is at least piecewise continuous on $\lambda \in\left[0,\|T\|^{2}\right]$, the operator $g_{\alpha}\left(T^{*} T\right)$ is wellposed.

Now we introduce two famous continuous regularization methods under the above framework, i.e., the Tikhonov regularization method and the TSVD method. In Tikhonov regularization method, the operator in the normal equation is perturbed to $T^{*} T+\alpha I$ and we solve

$$
\left(T^{*} T+\alpha I\right) x=T^{*} y^{\delta} .
$$

In fact in this case $g_{\alpha}(\lambda)$ can be expressed by

$$
g_{\alpha}(\lambda)=\frac{1}{\lambda+\alpha} .
$$

For the iterated Tikhonov method [13], we solve

$$
\left(T^{*} T+\alpha I\right) x_{m}=\alpha x_{m-1}+T^{*} y^{\delta},
$$

where $m$ is the iteration number, and $g_{\alpha}(\lambda)$ is

$$
g_{\alpha}(\lambda)=\frac{1}{\lambda}\left(1-\left(\frac{\alpha}{\alpha+\lambda}\right)^{m}\right) .
$$

In TSVD method, the singular values are truncated when the singular value is less than $\alpha$. In this case, $g_{\alpha}(\lambda)$ has the following expression

$$
g_{\alpha}(\lambda)= \begin{cases}\frac{1}{\lambda}, & \lambda \geqslant \alpha, \\ 0, & \lambda<\alpha .\end{cases}
$$


In general, we can analyze continuous regularization methods under this functional framework.

We denote the solution $x^{\text {sol }}$ in (2.13) by $x_{\alpha}^{\delta}$, which represents this solution is depended on the regularized parameter $\alpha$ and the noise level $\delta$. Similarly, we denote by

$$
x_{\alpha}=g_{\alpha}\left(T^{*} T\right) T^{*} y^{\text {exa }},
$$

the solution under the regularized parameter $\alpha$ and the exact right-hand side $y^{\text {exa }}$. Then the error between $x_{\alpha}^{\delta}$ and $x^{\dagger}$ is divided into two parts:

$$
\left\|x_{\alpha}^{\delta}-x^{\dagger}\right\| \leqslant\left\|x_{\alpha}^{\delta}-x_{\alpha}\right\|+\left\|x_{\alpha}-x^{\dagger}\right\| .
$$

In the right-hand side of (2.20), the first term is generated by the noise and the illposedness of the problem. The second term is generated by regularization.

For the second term in the right-hand side of (2.20), we have

$$
\begin{aligned}
x^{\dagger}-x_{\alpha} & =x^{\dagger}-g_{\alpha}\left(T^{*} T\right) T^{*} y^{\text {exa }} \\
& =\left(1-g_{\alpha}\left(T^{*} T\right) T^{*} T\right) x^{\dagger} \\
& =r_{\alpha}\left(T^{*} T\right) x^{\dagger},
\end{aligned}
$$

where

$$
r_{\alpha}(\lambda)=1-\lambda g_{\alpha}(\lambda)
$$

In the following discussion, we always assume $g_{\alpha}(\lambda)$ and $r_{\alpha}(\lambda)$ satisfy the following two properties [4]:

Assumption 2.1. On $\lambda \in\left[0,\|T\|^{2}\right], g_{\alpha}(\lambda)$ and $r_{\alpha}(\lambda)$ satisfy:

$$
\left\{\begin{array}{l}
\left|\lambda g_{\alpha}(\lambda)\right| \leqslant 1 \\
\left|g_{\alpha}(\lambda)\right| \leqslant \frac{1}{\alpha} \\
\left|r_{\alpha}(\lambda)\right| \leqslant 1
\end{array}\right.
$$

Assumption 2.2. On $\lambda \in\left[0,\|T\|^{2}\right], r_{\alpha}(\lambda)$ satisfies:

$$
\left|\lambda^{\mu} r_{\alpha}(\lambda)\right| \leqslant \alpha^{\mu} .
$$

When estimating the first term in the right-hand side of (2.20), we assume Assumption 2.1 is satisfied. And when estimating the second term in the right-hand side of (2.20), we assume Assumption 2.2 is satisfied.

Remark 2.1. It can be verified that Assumption 2.1 is always satisfied both for the Tikhonov method and the TSVD method. For the Tikhonov method with $\mu \leqslant 1$ and the TSVD method, Assumption 2.2 is also satisfied. Assumption 2.2 is often called the qualification of the regularization method $[22,24]$. For general source conditions, the related topic can be found in $[4,35]$. 
The following three Propositions are the main analysis of convergence in continuous regularization methods. For the mixed regularization method proposed in Section 3, the similar framework will be used to analyze the convergence.

Proposition 2.1. When Assumption 2.1 is satisfied, then we have

$$
\left\|x_{\alpha}-x_{\alpha}^{\delta}\right\| \leqslant \delta \sqrt{\frac{1}{\alpha}}
$$

Proposition 2.2. When Assumption 2.2 is satisfied, for $x^{\dagger} \in X_{\mu, \rho}$ we have

$$
\left\|x^{\dagger}-x_{\alpha}\right\| \leqslant \alpha^{\mu} \rho .
$$

The proofs of Proposition 2.1 and Proposition 2.2 can be found in [4, 20, 24]. Summarizing the two propositions above, we obtain

Proposition 2.3. When Assumptions 2.1 and 2.2 are satisfied, for $x^{\dagger} \in X_{\mu, \rho}$ we have

$$
\begin{aligned}
\left\|x_{\alpha}^{\delta}-x^{\dagger}\right\| & \leqslant\left\|x_{\alpha}^{\delta}-x_{\alpha}\right\|+\left\|x_{\alpha}-x^{\dagger}\right\| \\
& \leqslant \delta \sqrt{\frac{1}{\alpha}}+\alpha^{\mu} \rho .
\end{aligned}
$$

Now if we choose $\alpha$ such that

$$
c_{1}\left(\frac{\delta}{\rho}\right)^{\frac{2}{2 \mu+1}} \leqslant \alpha \leqslant c_{2}\left(\frac{\delta}{\rho}\right)^{\frac{2}{2 \mu+1}},
$$

we obtain

$$
\left\|x_{\alpha}^{\delta}-x^{\dagger}\right\| \leqslant c \delta^{\frac{2 \mu}{2 \mu+1}} \rho^{\frac{1}{2 \mu+1}},
$$

then the method associated with $\alpha$ reaches optimal order.

\subsection{Iterative regularization methods}

The typical well-known iterative regularization methods are the standard Landweber method and the CG method. Their algorithms may refer to $[4,20]$. There is no continuous regularization parameter in iterative regularization methods. Unlike the well-posed problems, iterative regularization methods for ill-posed problems are semi-convergent. That is to say, the iterative solution goes to the exact solution in first several iterations, then it goes away from the exact solution after a certain iteration. Therefore, some suitable stopping rules are required. One popular stopping rule is the discrepancy principle $[3,27,33,41]$

$$
\left\|y^{\delta}-T x_{k}^{\delta}\right\| \leqslant \tau \delta
$$

where $\tau$ is a parameter and $1<\tau<2$. Another typical stopping rule is the the monotone error rule (i.e., the ME-rule) $[7,8]$. The advantage of the ME-rule is that there is no need 
to fix the additional parameter $\tau$. The CG method is widely used in well-posed problems, and it also can be applied to ill-posed problems. In fact, in our algorithm we have applied the CG method to the normal equation of (2.7)

$$
T^{*} T x=T^{*} y^{\delta},
$$

and both the discrepancy principle and the ME-rule are used in our numerical computations to stop the iteration.

It can be proven that both the Landweber method and the CG method are with optimal order $[4,20,25]$. Although the iterative solution $x^{\text {it }}$ reaches optimal order, we will show that the solution can be improved in most situations in the next section. Improvements can be expected in our mixed regularization method.

\section{Mixed regularization methods}

\subsection{Mixed algorithm and optimal convergence order}

After (2.7) has been solved by an iterative regularization method, there is still a residual term $y^{\delta}-T x^{\text {it }}$. In order to solve the problem more precisely, we pay attention on the residual equation

$$
T x=y^{\delta}-T x^{\mathrm{it}} .
$$

To solve (3.1), a continuous regularization method is applied. The final solution is the summation of the solutions of the iterative regularization method and the continuous regularization method. More concretely, we have the following algorithm:

\section{Algorithm 3.1 Mixed regularization method}

1 To solve (2.7), first we obtain an iterative solution $x^{\text {it }}$ by solving its normal equation (2.30) with a iterative regularization method. Then we use a continuous regularization method with the function $g_{\alpha}(\lambda)$ to solve the residual equation (3.1):

$$
T x=y^{\delta}-T x^{\mathrm{it}} .
$$

2 The solution of (3.1) can be written as $g_{\alpha}\left(T^{*} T\right) T^{*}\left(y^{\delta}-T x^{\mathrm{it}}\right)$, therefore

$$
x^{\mathrm{mix}}=x^{\mathrm{it}}+g_{\alpha}\left(T^{*} T\right) T^{*}\left(y^{\delta}-T x^{\mathrm{it}}\right)
$$

is the final solution of this algorithm.

Note that Algorithm 3.1 is a general framework. Any continuous regularization method and any iterative regularization method can be combined together to yield a mixed regularization method. Hence the Algorithm 3.1 can be expected to possess wide application.

Now we analyse the convergence of Algorithm 3.1. In Algorithm 3.1, the error between the numerical solution and the exact solution is $\left\|x^{\dagger}-x^{\mathrm{mix}}\right\|$. We hope Algorithm 3.1 performs better than continuous regularization methods or iterative regularization methods 
when they are used separately. In fact, this can be guaranteed by the following theoretical analysis. First we prove the following lemma which is crucial in our theoretical framework.

Lemma 3.1. Suppose Assumption 2.1 is satisfied for $g_{\alpha}$ in Algorithm 3.1, then

$$
\left\|x^{\dagger}-x^{\mathrm{mix}}\right\| \leqslant\left\|x^{\dagger}-x^{\mathrm{it}}\right\|+\delta \sqrt{\frac{1}{\alpha}} .
$$

Proof. First we have

$$
\begin{aligned}
x^{\dagger}-x^{\mathrm{mix}}= & x^{\dagger}-x^{\mathrm{it}}-g_{\alpha}\left(T^{*} T\right) T^{*}\left(y^{\delta}-T x^{\mathrm{it}}\right) \\
= & x^{\dagger}-x^{\mathrm{it}}-g_{\alpha}\left(T^{*} T\right) T^{*}\left(y^{\text {exa }}-T x^{\mathrm{it}}\right) \\
& \quad+g_{\alpha}\left(T^{*} T\right) T^{*} y^{\text {exa }}-g_{\alpha}\left(T^{*} T\right) T^{*} y^{\delta} .
\end{aligned}
$$

Note that $g_{\alpha}\left(T^{*} T\right) T^{*} y^{\text {exa }}$ and $g_{\alpha}\left(T^{*} T\right) T^{*} y^{\delta}$ are just $x_{\alpha}$ and $x_{\alpha}^{\delta}$ respectively in Proposition 2.1. By Proposition 2.1,

$$
\left\|g_{\alpha}\left(T^{*} T\right) T^{*} y^{\text {exa }}-g_{\alpha}\left(T^{*} T\right) T^{*} y^{\delta}\right\| \leqslant \delta \sqrt{\frac{1}{\alpha}} .
$$

Therefore, by (3.5) and (3.6), we have

$$
\left\|x^{\dagger}-x^{\mathrm{mix}}\right\| \leqslant\left\|x^{\dagger}-x^{\mathrm{it}}-g_{\alpha}\left(T^{*} T\right) T^{*}\left(y^{\mathrm{exa}}-T x^{\mathrm{it}}\right)\right\|+\delta \sqrt{\frac{1}{\alpha}} .
$$

Additionally, we have

$$
\begin{aligned}
& x^{\dagger}-x^{\mathrm{it}}-g_{\alpha}\left(T^{*} T\right) T^{*}\left(y^{\mathrm{exa}}-T x^{\mathrm{it}}\right) \\
= & x^{\dagger}-x^{\mathrm{it}}-g_{\alpha}\left(T^{*} T\right) T^{*}\left(T x^{\dagger}-T x^{\mathrm{it}}\right) \\
= & \left(1-g_{\alpha}\left(T^{*} T\right) T^{*} T\right)\left(x^{\dagger}-x^{\mathrm{it}}\right) \\
= & r_{\alpha}\left(T^{*} T\right)\left(x^{\dagger}-x^{\mathrm{it}}\right),
\end{aligned}
$$

thus

$$
\begin{aligned}
& \quad\left\|x^{\dagger}-x^{\mathrm{it}}-g_{\alpha}\left(T^{*} T\right) T^{*}\left(y^{\mathrm{exa}}-T x^{\mathrm{it}}\right)\right\| \\
& \leqslant \sup _{\substack{\lambda \in\left[0,\|T\|^{2}\right] \\
\leqslant}}\left|r_{\alpha}(\lambda)\right| \cdot\left\|x^{\dagger}-x^{\mathrm{it}}\right\| \\
& \leqslant\left\|x^{\dagger}-x^{\mathrm{it}}\right\| .
\end{aligned}
$$

Finally, by (3.7) and (3.8), we obtain

$$
\left\|x^{\dagger}-x^{\mathrm{mix}}\right\| \leqslant\left\|x^{\dagger}-x^{\mathrm{it}}\right\|+\delta \sqrt{\frac{1}{\alpha}}
$$

which is just (3.4).

Lemma 3.1 is a basic estimate of $\left\|x^{\dagger}-x^{\mathrm{mix}}\right\|$. From Lemma 3.1, we arrive at the following optimal convergence theorem, i.e., Theorem 3.1. 
Theorem 3.1. Assume the iterative method in Algorithm 3.1 is with optimal order (e.g., the standard Landweber method and the CG method), and Assumption 2.1 is satisfied for $g_{\alpha}$ in Algorithm 3.1. Then for $\alpha \geqslant c(\delta / \rho)^{\frac{2}{2 \mu+1}}$, the mixed regularization method in Algorithm 3.1 is with optimal order.

Proof. Because of the optimal order of the iterative method in Algorithm 3.1, we have

$$
\left\|x^{\dagger}-x^{\mathrm{it}}\right\| \leqslant c_{1} \delta^{\frac{2 \mu}{2 \mu+1}} \rho^{\frac{1}{2 \mu+1}}
$$

Therefore, by Lemma 3.1, for $\alpha \geqslant c(\delta / \rho)^{\frac{2}{2 \mu+1}}$ we obtain

$$
\begin{aligned}
\left\|x^{\dagger}-x^{\mathrm{mix}}\right\| & \leqslant\left\|x^{\dagger}-x^{\mathrm{it}}\right\|+\delta \sqrt{\frac{1}{\alpha}} \\
& \leqslant c_{1} \delta^{\frac{2 \mu}{2 \mu+1}} \rho^{\frac{1}{2 \mu+1}}+c_{2} \delta^{\frac{2 \mu}{2 \mu+1}} \rho^{\frac{1}{2 \mu+1}} \\
& \leqslant c_{3} \delta^{\frac{2 \mu}{2 \mu+1}} \rho^{\frac{1}{2 \mu+1}},
\end{aligned}
$$

where $c_{1}, c_{2}$ and $c_{3}$ are constants. (3.11) indicates that the solution $x^{\text {mix }}$ is with optimal order.

Remark 3.1. When the continuous regularization method is applied separately, Assumption 2.2 is required. Moreover, by Proposition 2.3, the optimal order can reach only when $\alpha$ satisfies

$$
c_{1}\left(\frac{\delta}{\rho}\right)^{\frac{2}{2 \mu+1}} \leqslant \alpha \leqslant c_{2}\left(\frac{\delta}{\rho}\right)^{\frac{2}{2 \mu+1}} .
$$

In our mixed regularization method, Assumption 2.2 is not necessary and the optimal order can reach for a wider range of $\alpha$. For instance, the Tikhonov regularization method with $\mu>1$ does not satisfy Assumption 2.2, thus it can not reach the optimal order. However, it can reach optimal order in our mixed regularization method. We remark that for the iterated Tikhobov method, Theorem 3.1 still holds only $c$ is substituted by $\mathrm{cm}$. Here $m$ is the iteration number in the iterated Tikhobov method.

Remark 3.2. The wider range of $\alpha$ in Theorem 3.1, i.e. $\alpha \geqslant c(\delta / \rho)^{\frac{2}{2 \mu+1}}$, allows as to choose a more flexible $\alpha$. The choice of $\alpha$ is important in continuous regularization methods. Sometimes tiny variation of $\alpha$ could cause large variation in the error. Theorem 3.1 tells us we can choose a more larger $\alpha$. In the numerical computations in Section 4, we see that no matter how big $\alpha$ is, the error is almost on the same level with the optimal one. On the contrary, the large $\alpha$ usually causes large error when the continuous regularization method is applied separately to solve the problem. We remark that although the mixed regularization has this advantage it is still useful and significant to choose a proper regularization parameter in the second stage of the mixed method. 


\subsection{Mixed regularization method with the ME-rule}

When the noise level $\delta$ is known, we can choose the regularization parameter by a posterior method such as the ME-rule $[7,8]$. The ME-rule chooses the smallest regularization parameter $\alpha=\alpha_{M E}$ for which we can guarantee the ME-property: the error $\left\|x^{s o l}-x^{\dagger}\right\|$ is monotonically increasing for $\alpha \in\left[\alpha_{M E}, \infty\right)$. For the mixed regularization method, this means

$$
\frac{d}{d \alpha}\left\|x_{\alpha}+x^{i t}-x^{\dagger}\right\|^{2} \geq 0, \quad \forall \alpha \in\left[\alpha_{M E}, \infty\right)
$$

where $x^{i t}$ is the iterative solution in the first stage, and $x_{\alpha}=g_{\alpha}\left(T^{*} T\right) T^{*} y^{\delta, i t}$ is the solution by continuous parameter regularization methods in the second stage. Assuming $x_{\alpha}=$ $T^{*} w_{\alpha}$ and $z_{\alpha}:=\frac{d}{d \alpha} w_{\alpha}$, in order to guarantee the property (3.13) we make the following estimation under the condition $\left\|y^{\delta}-T x^{\dagger}\right\| \leqslant \delta$ :

$$
\begin{aligned}
& \frac{1}{2} \frac{d}{d \alpha}\left\|x_{\alpha}+x^{i t}-x^{\dagger}\right\|^{2}=\left(x_{\alpha}+x^{i t}-x^{\dagger}, T^{*} z_{\alpha}\right) \\
= & \left(T x_{\alpha}+T x^{i t}-y^{\delta}+y^{\delta}-T x^{\dagger}, z_{\alpha}\right) \geqslant\left(T x_{\alpha}-y^{\delta, i t}, z_{\alpha}\right)-\delta\left\|z_{\alpha}\right\|,
\end{aligned}
$$

and we solve the largest $\alpha=\alpha_{M E}$ satisfying

$$
\frac{\left(T x_{\alpha}-y^{\delta, i t}, z_{\alpha}\right)}{\left\|z_{\alpha}\right\|}=\delta,
$$

where $y^{\delta, i t}:=y^{\delta}-T x^{i t}$ is the residual from the first stage. We remark that (3.15) has a solution $\alpha$ if the following condition [36]

$$
\left\|P\left(T x_{m, \alpha}-y^{\delta, i t}\right)\right\|<\delta<\left\|T x_{m, \alpha}-y^{\delta, i t}\right\|
$$

is satisfied. Here $P$ is the orthoprojection of $Y$ onto $\mathscr{N}\left(A^{*}\right)$. We also remark that if in the second phase of regularization the $m$ times iterated Tikhonov approximation $x_{m, \alpha}$ in (2.16) ( $m=1$ in (2.14) ) is used, then $z_{\alpha}=T x_{m+1, \alpha}-y^{\delta, i t}$. In the case of nonexistence of solution to (3.15), i.e., (3.16) is violated, $\alpha_{M E}=\infty$ and in fact the second phase of regularization is cancelled. In numerical computations, we will see the occurrence of this phenomenon. Therefore, we propose the following new Algorithm 3.2:

Algorithm 3.2 Mixed regularization with the ME-rule

The solution $\alpha$ to (3.15) includes two cases:

Case 1: If $\alpha$ does not exist, then the solution $x^{\text {mix }}$ by the mixed regularization method is $x^{m i x}=x^{i t}$.

Case 2: If $\alpha$ exists, then the solution $x^{m i x}$ by the mixed regularization method is $x^{m i x}=$ $x^{i t}+x_{\alpha_{M E}}$.

Based on the algorithm above, we have the following result: 
Theorem 3.2. When Case 2 in Algorithm 3.2 is satisfied, the solution $x^{\text {mix }}$ obtained by the mixed regularization method is better than $x^{i t}$ by the iterative regularization method in the first stage.

Proof. Since

$$
\begin{aligned}
& x^{i t}=\lim _{\alpha \rightarrow \infty}\left(x_{\alpha}+x^{i t}\right) \\
& \frac{d}{d \alpha}\left\|x_{\alpha}+x^{i t}-x^{\dagger}\right\|^{2} \geqslant 0, \quad \forall \alpha \in\left[\alpha_{M E}, \infty\right),
\end{aligned}
$$

then we have

$$
\left\|x_{\alpha_{M E}}+x^{i t}-x^{\dagger}\right\| \leqslant\left\|x_{\infty}+x^{i t}-x^{\dagger}\right\|=\left\|x^{i t}-x^{\dagger}\right\|,
$$

which means the solution $x^{\text {mix }}$ by the mixed regularization method with the ME-rule is better than $x^{i t}$ by the iterative regularization method in the first stage.

Note that $\left\|x^{\dagger}-x^{\mathrm{it}}\right\|$ is the error in the corresponding iterative regularization method. Theorem 3.2 shows that using Algorithm 3.2 we can improve the solution from the iterative regularization method in the first stage. This is also confirmed by the numerical computations in Section 4. And in most situations the improvement is obvious. Note that for the (iterative) Tikhonov methods, (3.15) becomes [7]

$$
\frac{\left(\rho_{m, \alpha}, \rho_{m+1, \alpha}\right)}{\left\|\rho_{m+1, \alpha}\right\|}=\delta
$$

where $\rho_{m, \alpha}:=y^{\delta, i t}-T x_{m, \alpha}$.

Theorem 3.2 indicates that the mixed regularization method presented in this paper is effective theoretically. It behaves more precisely than the iterative regularization method. Next we will give some numerical tests to illustrate the theoretical aspects.

\section{Numerical computations}

The Fredholm integral equation of the first kind is a typical ill-posed problem. It arises in many applications in science and technology. For example, the gravity prospecting in geophysics is essential to solve the integral equation of the first kind. In this section, in order to illustrate our new algorithm, we solve three examples numerically by three different algorithms and compare the corresponding results.

The Fredholm integral equation of the first kind with the kernel $\kappa(s, t)$ can be written as:

$$
y(t)=T x(t)=\int_{a}^{b} \kappa(s, t) x(s) \mathrm{d} s, \quad \forall c \leqslant t \leqslant d .
$$

In the following we obtain three different examples by choosing the different kernels and the parameters $a, b, c, d$ in (4.1). 
Example 4.1. We choose $a=c=0, b=d=1$, and the integral kernel is defined as

$$
\kappa(s, t)= \begin{cases}0, & t \leqslant s \\ \frac{\mathrm{e}^{-\frac{1}{4(t-s)}}}{\sqrt{\pi(t-s)}}, & t>s .\end{cases}
$$

The exact solution $x^{\dagger}$ is chosen as

$$
x^{\dagger}(s)= \begin{cases}0, & s<0.25, \text { or } s>0.75 \\ 1, & 0.25 \leqslant s \leqslant 0.75\end{cases}
$$

then the exact right-hand side $y^{\text {exa }}(t)=f(0.75-t)-f(0.25-t)$, where

$$
f(t)= \begin{cases}0, & t \leqslant 0, \\ \frac{2}{\sqrt{\pi}}\left(\sqrt{t} \mathrm{e}^{-1 /(4 t)}-\int_{1 /(2 \sqrt{t})}^{\infty} \mathrm{e}^{\left.-z^{2} \mathrm{~d} z\right),}\right. & t>0 .\end{cases}
$$

Example 4.2. We choose $a=c=-6, b=d=6$, and the exact solution $x^{\dagger}$ is defined as

$$
x^{\dagger}(s)= \begin{cases}0, & |s| \geqslant 3 \\ 1+\cos \left(\frac{\pi}{3} s\right), & |s|<3\end{cases}
$$

We choose

$$
\kappa(s, t)=x^{\dagger}(t-s)
$$

and the exact right-hand side is

$$
y^{\text {exa }}(t)=(6-|t|)\left(1+\frac{1}{2} \cos \left(\frac{\pi}{3} t\right)\right)+\frac{9}{2 \pi} \sin \left(\frac{\pi}{3}|t|\right) .
$$

Example 4.3. We choose $a=c=0, b=d=\pi$, and the integral kernel is defined as

$$
\kappa(s, t)=\mathrm{e}^{t \cos (s)} .
$$

The exact solution $x^{\dagger}$ is

$$
x^{\dagger}(s)=\sin (s)
$$

and the exact right-hand side is

$$
y^{\operatorname{exa}}(t)=\frac{2 \sinh (t)}{t} .
$$

In the discretization we use the mesh size $2^{10}=1024$. We compute numerical solutions $x^{\text {sol }}$ with the following three different algorithms, named Algorithm I, II and III, and compute their corresponding $L^{2}$ norm of the errors, i.e. $\left\|x^{\text {sol }}-x^{\dagger}\right\|$.

- Algorithm I: Using the Tikhonov regularization method separately. The numerical solution is denoted by $x^{\text {Tik; }}$ 
Table 1: $L^{2}$ errors obtained by Algorithms I, II and III respectively for Example 4.1 with exact data.

\begin{tabular}{|c|c|c|c|}
\hline$\alpha$ & $\left\|x^{\text {Tik }}-x^{\dagger}\right\|$ & $\left\|x^{c g}-x^{\dagger}\right\|$ & $\left\|x^{\operatorname{mix}}-x^{\dagger}\right\|$ \\
\hline $10^{-7}$ & $8.9920 \mathrm{e}-02$ & $N_{\mathrm{i} t}=30, \quad 8.6525 \mathrm{e}-02$ & $5.9438 \mathrm{e}-02$ \\
\hline $10^{-8}$ & $7.7390 \mathrm{e}-02$ & $N_{\mathrm{it} t}=50, \quad 7.6598 \mathrm{e}-02$ & $5.9410 \mathrm{e}-02$ \\
\hline $10^{-9}$ & $6.7789 \mathrm{e}-02$ & $N_{\mathrm{i} t}=100,6.5674 \mathrm{e}-02$ & $5.9166 \mathrm{e}-02$ \\
\hline $10^{-10}$ & $6.0214 \mathrm{e}-02$ & $N_{\mathrm{i} t}=150,6.1940 \mathrm{e}-02$ & $5.7662 \mathrm{e}-02$ \\
\hline $10^{-11}$ & $5.4094 \mathrm{e}-02$ & $N_{\mathrm{i} t}=200,5.9441 \mathrm{e}-02$ & $5.3711 \mathrm{e}-02$ \\
\hline
\end{tabular}

- Algorithm II: Using the CG iterative method separately. The numerical solution is denoted by $x^{\mathrm{cg}}$;

- Algorithm III: Using the mixed regularization method. i.e. Algorithm 3.1. We apply the CG iterative method as the iterative regularization method and the Tikhonov regularization method as the continuous regularization method. The numerical solution is denoted by $x^{\text {mix }}$.

\subsection{Exact data}

In this section, we consider the case of exact data. Namely, we do not add the noise to right-hand side $y^{\text {ext }}$. There only exit the discretization errors in numerical computations. We remark Algorithm I and Algorithm III requires the regularization parameter $\alpha$. We choose a series of regularization parameters $\alpha$ and compute the $L^{2}$ errors. The results are listed in Tables 1-3 for Examples 4.1-4.3 respectively. For Algorithm II, since there is no noise in the data, the CG iteration is stopped at some fixed iteration step $N_{\mathrm{it}}$ and then the $L^{2}$ errors are yielded. The stopping iterations are set $N_{\mathrm{it}}=30,50,100,150$ and 200. In Tables 1-3, the solutions $x^{\text {mix }}$ in column 4 are obtained by using the values $x^{c g}$ at iteration number $N_{i t}=200$ in the first stage.

From Tables 1-3, we can see that the minimal $L^{2}$ errors for Algorithm I are 5.4094e-02, $1.8663 e-05$ and $2.3240 e-02$ while they are $5.3711 e-02,1.8784 e-06$ and $1.0666 e-02$ for Algorithm III. Obviously, Algorithm III behaves better than Algorithm I does. It is obvious that our mixed regularization method has better robustness to the regularization parameter $\alpha$ than the Algorithm I. From the third columns of Tables 1-3, we can see that the minimal $L^{2}$ errors obtained by Algorithm II are 5.9441e-02, 5.3048e- 05 and $1.0666 e-$ 02, which are not better than the corresponding results obtained by Algorithm III obviously.

We remark that in the case of exact data errors in Tikhonov method are monotonically decreasing for decreasing $\alpha$ values and the optimal $\alpha$ is zero theoretically $[7,8]$. However, this monotonicity can not be guaranteed due to discretization errors from numerical computations such as integral approximation. We can see this phenomenon in Table 2, which the errors in Tikhonov method for $\alpha=10^{-10}$ and $\alpha=10^{-11}$ are larger than for $\alpha=10^{-9}$. 
Table 2: $L^{2}$ errors obtained by Algorithms I, II and III respectively for Example 4.2 with exact data.

\begin{tabular}{||c|c|cc|c||}
\hline$\alpha$ & $\left\|x^{\text {Tik }}-x^{\dagger}\right\|$ & \multicolumn{2}{|c|}{$\left\|x^{\mathrm{cg}}-x^{\dagger}\right\|$} & $\left\|x^{\mathrm{mix}}-x^{\dagger}\right\|$ \\
\hline $10^{-7}$ & $8.8187 \mathrm{e}-05$ & $N_{\mathrm{i} t}=30$, & $5.2804 \mathrm{e}-04$ & $4.5452 \mathrm{e}-05$ \\
\hline $10^{-8}$ & $3.3808 \mathrm{e}-05$ & $N_{\mathrm{i} t}=50$, & $2.6486 \mathrm{e}-04$ & $2.8142 \mathrm{e}-05$ \\
\hline $10^{-9}$ & $1.8663 \mathrm{e}-05$ & $N_{\mathrm{i} t}=100$, & $1.0063 \mathrm{e}-04$ & $1.2512 \mathrm{e}-05$ \\
\hline $10^{-10}$ & $1.2356 \mathrm{e}-04$ & $N_{\mathrm{i} t}=150$, & $7.0209 \mathrm{e}-05$ & $4.9243 \mathrm{e}-06$ \\
\hline $10^{-11}$ & $1.1635 \mathrm{e}-03$ & $N_{\mathrm{i} t}=200$, & $5.3048 \mathrm{e}-05$ & $1.8784 \mathrm{e}-06$ \\
\hline
\end{tabular}

Table 3: $L^{2}$ errors obtained by Algorithms I, II and III respectively for Example 4.3 with exact data.

\begin{tabular}{||c|c|c|c||}
\hline$\alpha$ & $\left\|x^{\text {Tik }}-x^{\dagger}\right\|$ & $\left\|x^{\mathrm{cg}}-x^{\dagger}\right\|$ & $\left\|x^{\mathrm{mix}}-x^{\dagger}\right\|$ \\
\hline $10^{-7}$ & $4.1783 \mathrm{e}-02$ & $N_{\mathrm{i} t}=30,2.1799 \mathrm{e}-02$ & $1.0666 \mathrm{e}-02$ \\
\hline $10^{-8}$ & $3.8338 \mathrm{e}-02$ & $N_{\mathrm{i} t}=50,1.5000 \mathrm{e}-02$ & $1.0666 \mathrm{e}-02$ \\
\hline $10^{-9}$ & $3.0019 \mathrm{e}-02$ & $N_{\mathrm{i} t}=100,1.4376 \mathrm{e}-02$ & $1.0666 \mathrm{e}-02$ \\
\hline $10^{-10}$ & $2.4122 \mathrm{e}-02$ & $N_{\mathrm{i} t}=150,1.4323 \mathrm{e}-02$ & $1.0666 \mathrm{e}-02$ \\
\hline $10^{-11}$ & $2.3240 \mathrm{e}-02$ & $N_{\mathrm{i} t}=200,1.0666 \mathrm{e}-02$ & $1.0666 \mathrm{e}-02$ \\
\hline
\end{tabular}

\subsection{Noisy data with $\delta=10^{-4}$ and $\delta=10^{-2}$}

Now we turn to the case of adding noise. We add noise, which is uniformly distributed random error, to the exact right-hand side $y^{\text {exa }}$. The upper bound of noise is denoted by $\delta$, i.e.,

$$
\left\|y^{\text {exa }}-y^{\delta}\right\|:=\sqrt{\frac{1}{N_{t}+1} \sum_{i=0}^{N_{t}}\left(y_{i}^{\text {exa }}-y_{i}^{\delta}\right)^{2}}=\delta,
$$

where $N_{t}=1024$ is the discretization number. We consider two noise levels, i.e, $\delta=10^{-4}$ and $\delta=10^{-2}$. The noises added to the exact data are the random data following normal distribution with the mean square deviation $\delta$. The computational results are shown in Table 4 to Table 9. For Algorithm I and Algorithm III, we choose a series of regularization parameters and compute their $L^{2}$ errors which are listed in the second and fourth column of the tables respectively. For Algorithm II, two stopping rules, i.e., the ME-rule [7] and the discrepancy principle (DP) with $\tau=1.01$ are applied. In column 3 in Tables 4-9, the corresponding $\tau:=\left\|T x^{c g}-y^{\delta}\right\| / \delta$ and the number of iteration steps $n_{M E}$ according to the ME-rule are given in parentheses. Tables 4-6 are the results with noise level $\delta=10^{-4}$ for Examples 4.1-4.3 respectively while Tables 7-9 with noise level $\delta=10^{-2}$. From the results in Tables 4-9, we can clear see that the solutions $x^{\text {mix }}$ by the mixed regularization method are all better that the solutions $x^{c g}$ by the CG method. The mixed regularization method is more robust than the Tikhonov regularization method. The minimum errors for Tikhonov regularization method in Tables 4-6 are 8.3464e-02, $1.4673 e-03$ and $4.2458 e-02$ respectively while they are $8.3465 e-02,1.4276 e-03$ and $4.2449 e-02$ respectively for 
Table 4: $L^{2}$ errors obtained by Algorithms I, II and III respectively for Example 4.1 with $\delta=10^{-4}$.

\begin{tabular}{|c|c|c|c|}
\hline$\alpha$ & $\left\|x^{\mathrm{Tik}}-x^{\dagger}\right\|$ & $\left\|x^{\mathrm{cg}}-x^{\dagger}\right\|$ & $\left\|x^{\operatorname{mix}}-x^{\dagger}\right\|$ \\
\hline $10^{-2}$ & $3.7827 \mathrm{e}-01$ & \multirow{5}{*}{$\begin{array}{l}\text { 1.0855e-01 by ME-rule } \\
\left(\tau=1.0723, \quad n_{M E}=13\right)\end{array}$} & $1.0855 \mathrm{e}-01$ \\
\hline $10^{-5}$ & $1.3078 \mathrm{e}-01$ & & $1.0727 \mathrm{e}-01$ \\
\hline $5 \times 10^{-8}$ & $8.8707 \mathrm{e}-02$ & & $8.8516 \mathrm{e}-02$ \\
\hline $10^{-8}$ & $8.3464 \mathrm{e}-02$ & & $8.3465 \mathrm{e}-02$ \\
\hline $5 \times 10^{-9}$ & $8.5713 \mathrm{e}-02$ & & $8.5749 \mathrm{e}-02$ \\
\hline
\end{tabular}

Table 5: $L^{2}$ errors obtained by Algorithms I, II and III respectively for Example 4.2 with $\delta=10^{-4}$.

\begin{tabular}{|c|c|c|c|}
\hline$\alpha$ & $\left\|x^{\text {Tik }}-x^{\dagger}\right\|$ & $\left\|x^{c g}-x^{\dagger}\right\|$ & $\left\|x^{\text {mix }}-x^{\dagger}\right\|$ \\
\hline $10^{-2}$ & $1.0878 \mathrm{e}-02$ & \multirow{5}{*}{$\begin{array}{l}2.8784 e-03 \text { by ME-rule } \\
\left(\tau=1.0891, \quad n_{M E}=13\right)\end{array}$} & $2.8210 \mathrm{e}-03$ \\
\hline $10^{-4}$ & $1.7124 \mathrm{e}-03$ & & $1.5430 \mathrm{e}-03$ \\
\hline $6 \times 10^{-5}$ & $1.5233 \mathrm{e}-03$ & & $1.4411 \mathrm{e}-03$ \\
\hline $4 \times 10^{-5}$ & $1.4673 \mathrm{e}-03$ & & $1.4276 \mathrm{e}-03$ \\
\hline $2 \times 10^{-5}$ & $1.5634 \mathrm{e}-03$ & & $1.5594 \mathrm{e}-03$ \\
\hline
\end{tabular}

Table 6: $L^{2}$ errors obtained by Algorithms I, II and III respectively for Example 4.3 with $\delta=10^{-4}$.

\begin{tabular}{|c|c|c|c|}
\hline$\alpha$ & $\left\|x^{\mathrm{Tik}}-x^{\dagger}\right\|$ & $\left\|x^{\mathrm{cg}}-x^{\dagger}\right\|$ & $\left\|x^{\operatorname{mix}}-x^{\dagger}\right\|$ \\
\hline $10^{-3}$ & $1.1511 \mathrm{e}-01$ & \multirow{5}{*}{$\begin{array}{c}5.2643 e-02 \text { by ME-rule } \\
\left(\tau=1.0072, \quad n_{M E}=8\right)\end{array}$} & $5.2639 \mathrm{e}-02$ \\
\hline $10^{-6}$ & $4.9792 \mathrm{e}-02$ & & $4.9531 \mathrm{e}-02$ \\
\hline $10^{-7}$ & $4.2790 \mathrm{e}-02$ & & $4.2764 \mathrm{e}-02$ \\
\hline $5 \times 10^{-8}$ & $4.2458 \mathrm{e}-02$ & & $4.2449 \mathrm{e}-02$ \\
\hline $2 \times 10^{-8}$ & $4.4331 \mathrm{e}-02$ & & $4.4332 \mathrm{e}-02$ \\
\hline
\end{tabular}

the mixed regularization method. We can clearly see the mixed regularization method performs better.

Tables 7-9 are the results of $L^{2}$ errors for noise level $\delta=10^{-2}$ for Examples 4.1-4.3 respectively. And we can see the minimum error for Tikhonov regularization method in Tables $7-9$ are $1.6184 e-01,1.1357 e-02$ and $7.8204 e-02$ respectively while they are $1.5373 e-01,1.1114 e-02$ and $7.8199 e-02$ respectively for the mixed regularization method. Obviously, we get the same conclusion again like the case of noise level $\delta=10^{-4}$.

From Tables 2-6 we can see the effectiveness of the new mixed regularization method obviously. Firstly, we compare Algorithm II and Algorithm III. When $\alpha$ is large, the results of Algorithm III (i.e., column 2) are better than the iterative solutions of Algorithm II (i.e., column 4) obviously. In fact, these three tables show that the iterative solutions of Algorithm II is just the limitation of the solution of Algorithm III while $\alpha$ becomes big. The optimal $\alpha$ usually is not so big, thus a smaller $\alpha$ usually brings a better result than the 
Table 7: $L^{2}$ errors obtained by Algorithms I, II and III respectively for Example 4.1 with $\delta=10^{-2}$.

\begin{tabular}{|c|c|c|c|}
\hline$\alpha$ & $\left\|x^{\text {Tik }}-x^{\dagger}\right\|$ & $\left\|x^{\mathrm{cg}}-x^{\dagger}\right\|$ & $\left\|x^{\operatorname{mix}}-x^{\dagger}\right\|$ \\
\hline $10^{-2}$ & $3.7930 \mathrm{e}-01$ & \multirow{5}{*}{$\begin{array}{c}\text { 1.7793e-01 by ME-rule } \\
\left(\tau=1.0012, n_{M E}=4\right)\end{array}$} & $1.7745 \mathrm{e}-01$ \\
\hline $10^{-3}$ & $2.2166 \mathrm{e}-01$ & & $1.7399 \mathrm{e}-01$ \\
\hline $10^{-4}$ & $1.7450 \mathrm{e}-01$ & & $1.5958 \mathrm{e}-01$ \\
\hline $5 \times 10^{-5}$ & $1.6184 \mathrm{e}-01$ & & $1.5373 \mathrm{e}-01$ \\
\hline $10^{-5}$ & $1.6428 \mathrm{e}-01$ & & $1.6587 \mathrm{e}-01$ \\
\hline
\end{tabular}

Table 8: $L^{2}$ errors obtained by Algorithms I, II and III respectively for Example 4.2 with $\delta=10^{-2}$.

\begin{tabular}{|c|c|c|c|}
\hline$\alpha$ & $\left\|x^{\mathrm{Tik}}-x^{\dagger}\right\|$ & $\left\|x^{\mathrm{cg}}-x^{\dagger}\right\|$ & $\left\|x^{\mathrm{mix}}-x^{\dagger}\right\|$ \\
\hline 10 & $2.9421 \mathrm{e}-01$ & \multirow{5}{*}{$\begin{array}{c}2.1085 e-02 \text { by ME-rule } \\
\left(\tau=1.1432, \quad n_{M E}=4\right)\end{array}$} & $2.1031 \mathrm{e}-02$ \\
\hline 1 & $7.5369 \mathrm{e}-02$ & & $2.0782 \mathrm{e}-02$ \\
\hline $10^{-1}$ & $2.2814 \mathrm{e}-02$ & & $1.8696 \mathrm{e}-02$ \\
\hline $10^{-2}$ & $1.1357 \mathrm{e}-02$ & & $1.1114 \mathrm{e}-02$ \\
\hline $10^{-3}$ & $1.4457 \mathrm{e}-02$ & & $1.4467 \mathrm{e}-02$ \\
\hline
\end{tabular}

Table 9: $L^{2}$ errors obtained by Algorithms I, II and III respectively for Example 4.3 with $\delta=10^{-2}$.

\begin{tabular}{|c|c|c|c|}
\hline$\alpha$ & $\left\|x^{\text {Tik }}-x^{\dagger}\right\|$ & $\left\|x^{c g}-x^{\dagger}\right\|$ & $\left\|x^{\operatorname{mix}}-x^{\dagger}\right\|$ \\
\hline $10^{-3}$ & $1.1499 \mathrm{e}-01$ & \multirow{5}{*}{$\begin{array}{c}1.1326 e-01 \text { by ME-rule } \\
\left(\tau=9.9832 e-01, n_{M E}=4\right)\end{array}$} & $1.1200 \mathrm{e}-01$ \\
\hline $10^{-4}$ & $1.0344 \mathrm{e}-01$ & & $1.0329 \mathrm{e}-01$ \\
\hline $10^{-5}$ & $8.1882 \mathrm{e}-02$ & & $8.1816 \mathrm{e}-02$ \\
\hline $10^{-6}$ & $7.8204 \mathrm{e}-02$ & & 7.8199e-02 \\
\hline $10^{-7}$ & $1.6959 \mathrm{e}-01$ & & $1.6959 \mathrm{e}-01$ \\
\hline
\end{tabular}

limitation. Secondly, we compare Algorithm I and Algorithm III. Almost for all the same $\alpha$, the results in Algorithm III (i.e., column 4) are better than those of Algorithm I (i.e., column 1). Especially when $\alpha$ is bigger than the optimal one. This is in accordance with Theorem 3.1. Only in the case when the regularization parameter $\alpha$ is close to the optimal value the results of Algorithm I are comparable with those of Algorithm III. However, the optimal $\alpha$ is depending on problem and is usually hard to choose in many applications. Algorithm III allows us to choose a bigger $\alpha$. Moreover, the error for the big $\alpha$ is on the same order of magnitude as the optimal one, which is both confirmed by Theorem 3.1. Thirdly, the mixed regularization method is not sensitive to the regularization parameter like the continuous regularization method.

\subsection{Choosing regularization parameter based on the ME-rule}

In Section 4.1 and Section 4.2, we have chosen a group of regularization parameters and compared the solutions. In this subsection, we choose regularization parameter based 
Table 10: $L^{2}$ errors and regularization parameters $\alpha$ obtained based on the ME-rule for three examples.

\begin{tabular}{||c|c|c|c|c|c|c||}
\hline \multirow{2}{*}{ Exs. } & \multicolumn{3}{|c|}{$\delta=10^{-4}$} & \multicolumn{3}{c||}{$\delta=10^{-2}$} \\
\cline { 2 - 7 } & Tikhonov & CG stage & Mixed & Tikhonov & CG stage & Mixed \\
\hline \multirow{2}{*}{ Ex. 4.1 } & $9.53 \mathrm{e}-02$ & $1.09 \mathrm{e}-01$ & $9.55 \mathrm{e}-02$ & $1.77 \mathrm{e}-01$ & $1.78 \mathrm{e}-01$ & $1.73 \mathrm{e}-01$ \\
& $(2.00 \mathrm{e}-07)$ & $n_{M E}=13$ & $(2.64 \mathrm{e}-07)$ & $(1.16 \mathrm{e}-04)$ & $n_{M E}=4$ & $(8.01 \mathrm{e}-04)$ \\
\hline \multirow{2}{*}{ Ex. 4.2 } & $1.72 \mathrm{e}-03$ & $2.88 \mathrm{e}-03$ & $1.67 \mathrm{e}-03$ & $1.13 \mathrm{e}-02$ & $2.11 \mathrm{e}-02$ & $1.12 \mathrm{e}-02$ \\
& $(1.02 \mathrm{e}-04)$ & $n_{M E}=13$ & $(1.49 \mathrm{e}-04)$ & $(9.72 \mathrm{e}-03)$ & $n_{M E}=4$ & $(1.02 \mathrm{e}-02)$ \\
\hline \multirow{2}{*}{ Ex. 4.3 } & $4.47 \mathrm{e}-02$ & $5.26 \mathrm{e}-02$ & $4.47 \mathrm{e}-02$ & $1.12 \mathrm{e}-01$ & $1.13 \mathrm{e}-01$ & $1.13 \mathrm{e}-01$ \\
& $(2.18 \mathrm{e}-07)$ & $n_{M E}=8$ & $(2.18 \mathrm{e}-07)$ & $(5.67 \mathrm{e}-04)$ & $n_{M E}=4$ & $(\infty)$ \\
\hline
\end{tabular}

Table 11: $L^{2}$ errors and regularization parameters $\alpha$ obtained based on the ME-rule for three examples.

\begin{tabular}{||c|c|c|c|c|c|c||}
\hline \multirow{2}{*}{ Exs. } & \multicolumn{3}{|c|}{$\delta=10^{-4}$} & \multicolumn{3}{c||}{$\delta=10^{-2}$} \\
\cline { 2 - 7 } & Tikhonov & CG stage & Mixed & Tikhonov & CG stage & Mixed \\
\hline \multirow{2}{*}{ Ex. 4.1 $1^{\prime}$} & $3.00 \mathrm{e}-04$ & $7.04 \mathrm{e}-05$ & $7.04 \mathrm{e}-05$ & $5.73 \mathrm{e}-03$ & $3.41 \mathrm{e}-03$ & $3.41 \mathrm{e}-03$ \\
& $(3.73 \mathrm{e}-04)$ & $n_{M E}=2$ & $(\infty)$ & $(9.26 \mathrm{e}-03)$ & $n_{M E}=1$ & $(\infty)$ \\
\hline \multirow{2}{*}{ Ex. 4.2' } & $4.23 \mathrm{e}-04$ & $2.24 \mathrm{e}-04$ & $8.51 \mathrm{e}-05$ & $7.54 \mathrm{e}-03$ & $1.56 \mathrm{e}-03$ & $1.05 \mathrm{e}-03$ \\
& $(4.28 \mathrm{e}-04)$ & $n_{M E}=7$ & $(8.64 \mathrm{e}-03)$ & $(7.69 \mathrm{e}-03)$ & $n_{M E}=4$ & $(4.90 \mathrm{e}+00)$ \\
\hline \multirow{2}{*}{ Ex. 4.3' } & $2.34 \mathrm{e}-04$ & $3.25 \mathrm{e}-04$ & $7.45 \mathrm{e}-05$ & $4.98 \mathrm{e}-03$ & $5.51 \mathrm{e}-03$ & $8.45 \mathrm{e}-04$ \\
& $(3.35 \mathrm{e}-04)$ & $n_{M E}=4$ & $(1.64 \mathrm{e}-03)$ & $(6.83 \mathrm{e}-03)$ & $n_{M E}=2$ & $(5.77 \mathrm{e}-02)$ \\
\hline
\end{tabular}

Table 12: $L^{2}$ errors and regularization parameters $\alpha$ obtained based on the ME-rule for three examples.

\begin{tabular}{||c|c|c|c|c|c|c||}
\hline \multirow{2}{*}{ Exs. } & \multicolumn{3}{|c|}{$\delta=10^{-4}$} & \multicolumn{3}{c||}{$\delta=10^{-2}$} \\
\cline { 2 - 7 } & Tikhonov & CG stage & Mixed & Tikhonov & CG stage & Mixed \\
\hline \multirow{2}{*}{ Ex. 4.1' } & $1.36 \mathrm{e}-04$ & $7.042 \mathrm{e}-05$ & $7.04 \mathrm{e}-05$ & $4.18 \mathrm{e}-03$ & $3.41 \mathrm{e}-03$ & $3.41 \mathrm{e}-03$ \\
& $(1.43 \mathrm{e}-02)$ & $n_{M E}=2$ & $(\infty)$ & $(1.95 \mathrm{e}-01)$ & $n_{M E}=1$ & $(\infty)$ \\
\hline \multirow{2}{*}{ Ex. 4.2' } & $6.60 \mathrm{e}-05$ & $2.24 \mathrm{e}-04$ & $6.51 \mathrm{e}-05$ & $1.28 \mathrm{e}-03$ & $1.56 \mathrm{e}-03$ & $1.07 \mathrm{e}-03$ \\
& $(9.06 \mathrm{e}-02)$ & $n_{M E}=7$ & $(1.07 \mathrm{e}-01)$ & $(1.34 \mathrm{e}+00)$ & $n_{M E}=4$ & $(9.04 \mathrm{e}+01)$ \\
\hline \multirow{2}{*}{ Ex. 4.3' } & $4.73 \mathrm{e}-05$ & $3.25 \mathrm{e}-04$ & $4.41 \mathrm{e}-05$ & $3.23 \mathrm{e}-04$ & $5.51 \mathrm{e}-03$ & $3.23 \mathrm{e}-04$ \\
& $(2.15 \mathrm{e}-02)$ & $n_{M E}=4$ & $(2.15 \mathrm{e}-02)$ & $(8.01 \mathrm{e}-01)$ & $n_{M E}=2$ & $(8.40 \mathrm{e}-01)$ \\
\hline
\end{tabular}

on the ME-rule. In Table 10, the $L^{2}$ errors of the Tikhonov method and our mixed Tikhonov method for three examples (Ex. 4.1, Ex. 4.2 and Ex. 4.3) with two noise levels $\left(\delta=10^{-4}\right.$ and $10^{-2}$ ) are presented. There is no iteration in Tikhonov method. The number in parentheses in Table 10 is the regularization parameter according to the ME-rule. The errors of CG and the corresponding iteration number $n_{M E}$ according to the ME-rule are also listed in the third column and the sixth column in Table 10. In the case of noise level $\delta=10^{-4}$, the errors of the mixed Tikhonov method are smaller than those of the Tikhonov 
method for Example 4.2 and Example 4.3, and for Example 4.1 it is only very little bigger. In the case of noise level $\delta=10^{-2}$, the errors of the mixed Tikhonov method are all smaller than those of the Tikhonov method for the three examples.

Since the performance of methods and the ME-rule generally depends on the smoothness of $\mu$ of the exact solutions, we complemented the solutions $x^{\dagger}$ of three examples with smoothened solutions $\left(T^{*} T x^{\dagger}\right)$ and correspondingly compute the right-hand side as $T\left(T^{*} T\right) x^{\dagger}$. This operation has guaranteed the smoothness of solutions $\mu>1$. Corresponding to the original three examples, we name the resulting three examples as Ex. 4.1', Ex. $4.2^{\prime}$ and Ex. 4.3' respectively. The computational results of $L^{2}$ errors are shown in Table 11 and Table 12. The difference between Table 11 and Table 12 is that there is no iteration in Tikhonov method ( $m=1$ ) in Table 11 while the iterated Tikhonov method is adopted $(m=10)$ in Table 12. Similarly, in Table 11 and Table 12 the number in parentheses is the regularization parameters. And the errors of CG and the corresponding iteration number $n_{M E}$ according to the ME-rule are also listed in the third column and the sixth column. Comparisons show that the $L^{2}$ errors of the mixed regularization method are much smaller than those of the Tikhonov method both in Table 11 and Table 12. Thus the advantage of the mixed regularization method is more obvious if the solution is more smoother. We remark that the larger errors in the Tikhonov method than the mixed method are due to the fact that the Tikhonov method is order optimal only for the smoothness index $\mu \leq 1$. Moreover, we can see the iterated Tikhonov method $(m=10)$ in Table 12 performs better than the non-iterated Tikhonov method $(m=1)$ in Table 11.

\section{Conclusions}

We have described a new mixed regularization method for ill-posed problems. It is a combination of iterative regularization methods and continuous regularization methods. The iterative regularization methods do not need continuous regularization parameters while the continuous regularization methods do. The mixed regularization method can be considered as an improvement of the continuous regularization method. In continuous regularization methods, the choice of the regularization parameter is crucial because the solution depends on the parameter sensitively. Our theoretical analysis shows that the new mixed regularization method can reduce the sensitivity of regularization parameter and improve the solution of iterative regularization methods. It can reach the optimal convergence order under a much wider range of the parameter than the continuous regularization method. Moreover, our mixed regularization method is a general algorithm framework. Any iterative regularization method and continuous regularization method can be combined together. The numerical experiments illustrate that the mixed regularization method is more effective than continuous regularization methods or iterative regularization methods, which fits to our theoretical analysis. Some further theoretical analysis will be our future research topic.

Acknowledgments We appreciate the anonymous reviewers very much for their very valuable comments and helps. This research is supported by the National Natural Science 
Foundation of China under the grant number 11471328. It is also partially supported by the National Center for Mathematics and Interdisciplinary Sciences, Chinese Academy of Sciences.

\section{References}

[1] P. Craven and G. Wahba, Smoothing noisy data with spline functions-estimating the correct degree of smoothing by the method of generalized cross-validation, Numer. Math., 31 (1979), pp. 377-403.

[2] H. Egger and A. Neubauer, Preconditioning Landweber iteration in Hilbert scales, Numer. Math., 101 (2005), pp. 643-662.

[3] H. W. ENGL, Discrepancy principles for Tikhonov regularization of ill-posed problems leading to optimal convergence rates, J. Optimiz. Theory App., 52(2) (1987), pp. 209-215.

[4] H. W. Engl, M. Hanke And A. Neubauer, Regularization of Inverse Problems, Kluwer, Dordrecht, 1996.

[5] S. F. GiLYAzov, Regularizing conjugate-direction methods, Comp. Math. Math. Phys., 35(4) (1995), pp. 385-394.

[6] G. H. Golub, M. Heath And G. Wahba, Generalized cross-validation as a method for choosing a good ridge parameter, Technometrics, 21(2) (1979), pp. 215-223.

[7] U. Hämarik, U. Kangro, R. Palm, T. Raus and U. TAutenhahn, Monotonicity of error of regularized solution and its use for parameter choice, Inverse Probl. Sci. En., 22(1) (2014), pp. 10-30.

[8] U. HäMARIK, On comparison of accuracy of approximate solutions of operator equations with noisy data, AIP Conf. Proc., 1783 (2015), 480042-1-480042-4.

[9] M. HANKE, Accelerated Landweber iterations for the solution of ill-posed equations, Numer. Math., 60 (1991), pp. 341-373.

[10] M. HANKE, The minimal error conjugate-gradient method is a regularization method, Proceedings of the American Mathematical Society, 123(11) (1995), pp. 3487-3497.

[11] M. Hanke, Conjugate Gradient Type Methods for Ill-posed Problems, Longman Scientific and Technical, Wiley, 1995.

[12] M. HANKe, A. Neubauer, AND O. Scherzer, A convergence analysis of the Landweber iteration for nonlinear ill-posed problems, Numer. Math., 72 (1995), pp. 21-37.

[13] M. Hanke and C. W. Groetsch, Nonstationary iterated Tikhonov regularization, J. Optim. Theory Appl., 98(1) (1998), pp. 37-53.

[14] P. C. HANSEn, Discrete Inverse Problems: Insight and Algorithms, SIAM, Philadelphia, 2010.

[15] P. C. HANSEN, Analysis of discrete ill-posed problems by means of the L-curve, SIAM Review, 34(4) (1992), pp. 561-580.

[16] P. C. HANSEN, AND D. P. O'LeARY, The use of the L-curve in the regularization of discrete ill-posed problems, SIAM J. Sci. Comput., 14(6) (1993), pp. 1487-1503.

[17] H. GFRERER, An a posteriori parameter choice for ordinary and iterated Tikhonov regularization of ill-posed problems leading to optimal convergence rates, Math. Comput., 49(180) (1987), pp. 507-522.

[18] Q. N. Jin, Applications of the modified discrepancy principle to Tikhonov regularization of nonlinear ill-posed problems, SIAM J. Numer. Anal., 36(2) (1999), pp. 475-490.

[19] J. T. King, Multilevel algorithms for ill-posed problems, Numer. Math., 61(1) (1992), pp. 311334.

[20] A. КiRch, An Introduction to the Mathematical Theory of Inverse Problems, Springer-Verlag, New York, 1996. 
[21] L. LANDWEBER, An iteration formula for Fredholm integral equations of the first kind, Am. J. Math., 73(3) (1951), pp. 615-624.

[22] S. Lu, And S. V. Pereverzev, Regularization Theory for Ill-posed Problems. Selected Topics, Am. Nat., 109(969) (2013), pp. 529-539.

[23] J. T. MARTI, An algorithm for computing minimum norm solutions of Fredholm integral equations of the first kind, SIAM J. Numer. Anal., 15(6) (1978), pp. 1071-1076.

[24] P. Mathé, and S. V. Pereverzev, Geometry of linear ill-posed problems in variable Hilbert scales, Inverse Probl., 19(3) (2003), pp. 789-803.

[25] A. S. NemirovskiI, The regularization properties of the adjoint gradient method in ill-posed problems, USSR Comput. Math. Math. Phys., 26(2) (1986), pp. 7-16.

[26] A. NeubAuer, On Landweber iteration for nonlinear ill-posed problems in Hilbert scales, Numer. Math., 85 (2000), pp. 309-328.

[27] R. Plato, On the discrepancy principle for iterative and parametric methods to solve linear illposed equations, Numer. Math., 75(1) (1996), pp. 99-120.

[28] R. PLATO, The method of conjugate residuals for solving the Galerkin equations associated with symmetric positive semidefinite ill-posed problems, SIAM J. Numer. Anal., 35(4) (1998), pp. 1621-1645.

[29] R. Plato, The conjugate gradient method for linear ill-posed problems with operator perturbations, Numer. Algorithms, 20(1) (1999), pp. 1-22.

[30] R. Ramlau, A modified Landweber method for inverse problems, Numer. Func. Anal. Opt., 20(12) (1999), pp. 79-98.

[31] L. Reichel, AND A. Shyshrov, Cascadic multilevel methods for ill-posed problems, J. Computat. Appl. Math., 233(5) (2010), pp. 1314-1325.

[32] O. SCHERZER, Convergence criteria of iterative methods based on Landweber iteration for solving nonlinear problems, J. Math. Anal. Appl., 194 (1995), pp. 911-933.

[33] E. Sсноск, Parameter choice by discrepancy principles for the approximate solution of ill-posed problems, Integr. Equat. Oper. Th., 7(6) (1984), pp. 895-898.

[34] U. TAutenhahn, On a general regularization scheme for nonlinear ill-posed problems, Inverse Probl., 13 (1997), pp. 1427-1437.

[35] U. Tautenhahn, Optimality for ill-posed problems under general source conditions, Numer. Funct. Anal. Optim., 19 (1998), pp. 377-398.

[36] U. TAUTENHAHN, AND U. HämARIK, The use of monotonicity for choosing the regularization parameter in ill-posed problems, Inverse Probl., 15 (1999), pp. 1487-1505.

[37] A. N. Tiкноnov, Solution of incorrectly formulated problems and regularization method, Doklady Akademii Nauk SSSR, 151 (1963), pp. 501-504.

[38] A. N. Tikнonov, Regularization of incorrectly posed problems, Dokl. Akad. Nauk SSSR, 153 (1963), pp. 49-52.

[39] A. N. Tikhonov, And V. Y. ARsenin, Solutions of Ill-posed Problems, Wiley, New York, 1977.

[40] A. N. TiKhonov, AND V. B. Glasko, On the approximate solution of Fredholm integral equations of the first kind, USSR Comput. Math. Math. Phys., 4 (1964), pp. 564-571.

[41] G. M. VAINIKKo, The discrepancy principle for a class of regularization methods, USSR Comput. Math. Math. Phys., 22 (1982), pp. 1-19.

[42] C. R. VogeL, Computational Methods for Inverse Problems, SIAM, Philadelphia, 2002.

[43] G. WAHBA, Practical approximate solutions of linear operator equations when the data are noisy, SIAM J. Numer. Anal., 14(4) (1977), pp. 651-667.

[44] J. XIE, AND J. Zou, An improved model function method for choosing regularization parameters in linear inverse problems, Inverse Probl., 18 (2002), pp. 631-643. 